\title{
UNIFORM SPACES OF COUNTABLE TYPE
}

\author{
GIOVANNI VIDOSSICH
}

\begin{abstract}
Uniform spaces of countable type are uniform spaces having a basis of countable uniform coverings. The present note investigates some of their properties. It is proved the existence of uniformly locally finite uniform refinements, the separability of some of their function spaces and that an injective space of countable type whose points are intersections of at most $2 \mathrm{~N}_{0}$ open sets must be separable.
\end{abstract}

A uniform space is said to be of countable type when it has a basis of countable uniform coverings. These spaces may be viewed among the simplest uniform spaces. Their properties find applications beyond Topology, as in [4] where they are used to prove the separability of suitable $L F$-spaces. Moreover, to each uniform space there is associated in a natural way a uniformity of countable type, which turns out to be compatible with the topology of the space.

The aim of this note is to investigate some of the properties of these spaces. We solve positively for them Isbell's problem $B_{3}$, we estimate the density character of some of their function spaces and we prove that an injective space of countable type, whose points are intersections of at most $2^{\aleph_{0}}$ open sets (for examples, the points may be $G_{\delta}$-sets), must be separable. This implies that a sum of injective space needs not be injective.

Terminology. We base our terminology on [2], so that our uniform spaces are all assumed to be Hausdorff.

We begin by recalling some well-known facts. In separable topological spaces as well as in Lindelöf spaces every compatible uniformity is o i countable type by [2, ii.33]. Every product of spaces of countable type is of countable type by [2, Chapter ii, Exercise 2]. By [2, Chapter iii, Exercise 2], the countable uniform coverings of any uniform space $\mu X$ form a basis for a uniformity $\nu$ on $X$ called the $\boldsymbol{\aleph}_{1 \text { - }}$ reflection of $\mu X ; \nu$ is compatible with the topology of $X$ since $\nu$ is less fine than $\mu$ and finer than the precompact reflection of $\mu$.

The following theorem asserts that Isbell's problem $B_{3}[2$, p. 144] has a positive solution for spaces of countable type.

Received by the editors October 23, 1969.

AMS Subject Classifications. Primary 5430; Secondary 5440, 5428.

Key Words and Phrases. Uniform space, uniform space of countable type, injective space, compact space, $\boldsymbol{\aleph}_{1}$-reflection, product and sum of uniform spaces, function space, uniform retraction, countable uniform covering, uniformly locally finite uniform covering, star-refinement. 
Theorem 1. A uniform space of countable type has a basis of (countable) uniformly locally finite uniform coverings.

Proof. Let $X$ be a uniform space of countable type, $u=\left\{U_{n} \mid n \in N\right\}$ a countable uniform cover of $X$, and $V=\left\{V_{n} \mid n \in N\right\}$ a countable uniform star-star-refinement of $\mathfrak{u}\left(=\mathcal{V}^{* *}\right.$ refines $\left.\mathcal{u}\right)$. Put

$$
W_{n}=\operatorname{St}\left(V_{n}, \mathcal{V}\right) \cap \bigcap_{i=0}^{n-1}\left(X \backslash \operatorname{St}\left(V_{i}, \mathcal{V}\right)\right) \quad(n \in N) .
$$

Choose $n \in N$. If $i>n$, then $W_{i} \subseteq X \backslash \operatorname{St}\left(V_{n}, \mathcal{V}\right)$, and hence $W_{i} \cap$ $\operatorname{St}\left(V_{n}, v\right)=\varnothing$. Therefore

$$
\left\{i \in N \mid W_{i} \cap \operatorname{St}\left(V_{n}, v\right) \neq \varnothing\right\} \subseteq\{0, \cdots, n\} .
$$

For any $x \in X$, the set $\left\{n \in N \mid x \in \operatorname{St}\left(V_{n}, \mathcal{V}\right)\right\}$ has a smallest element $n_{x}$, so that $x \in W_{n_{x}}$. This shows that $\left\{W_{n} \mid n \in N\right\}$ is a covering of $X$. Therefore $V$ refines $\mathscr{W}=\left\{\operatorname{St}\left(W_{n}, \mathcal{V}\right) \mid n \in N\right\}$, so that $\mathscr{W}$ is a uniform cover of $X$. Since $\operatorname{St}\left(W_{n}, \mathcal{V}\right) \subseteq \operatorname{St}\left(\operatorname{St}\left(V_{n}, v\right), v\right)$ and $v$ is a star-starrefinement of $\mathcal{U}, \mathcal{W}$ refines $\mathcal{u}$. Thus we have only to prove that $\mathcal{W}$ is uniformly locally finite. For, choose $V_{n} \in \mathcal{V}$. If $V_{n} \cap \operatorname{St}\left(W_{i}, \mathcal{V}\right) \neq \varnothing$, then $\operatorname{St}\left(V_{n}, \mathcal{V}\right) \cap W_{i} \neq \varnothing$. From (*) it follows $i \leqq n$, so that $V_{n}$ can meet only a finite number of elements of W. Q.E.D.

Lemma. Let $\left(X_{\alpha}\right)_{\alpha \in A}$ be a family of separable topological spaces and $Y$ a Hausdorff topological space in which every point is the intersection of at most $2 \boldsymbol{\aleph}_{0}$ open sets. Then for every continuous mapf: $\prod_{\alpha \in A} X_{\alpha} \rightarrow Y$ there is $B \subseteq A$ such that $\operatorname{Card}(B) \leqq 2^{\aleph_{0}}$ and $f=g \circ \pi_{B}$, where $\pi_{B}$ : $\prod_{\alpha \in A} X_{\alpha} \rightarrow \prod_{\alpha \in B} X_{\alpha}$ is the canonical projection and $g: \prod_{\alpha \in B} X_{\alpha} \rightarrow Y$ is continuous.

Proof. For each $x \in \prod_{\alpha \in A} X_{\alpha}, f(x)$ is the intersection of at most $2^{\boldsymbol{N}_{0}}$ open sets. Therefore $f^{-1}(f(x))$ has the same property, so that it contains an intersection of at most $2^{\aleph_{0}}$ basic open sets of the product containing $x$. Then there is $B_{x} \subseteq A$ such that $\operatorname{Card}\left(B_{x}\right) \leqq 2^{\aleph_{0}}$ and each point having the same coordinates in $B_{x}$ as $x$ has the same image under $f$. Moreover, $\prod_{\alpha \in B_{x}} X_{\alpha}$ is separable by [1, viii. 7.2. (3)]. Thus we may repeat exactly the proof of Gleason theorem [2, vii.19] to obtain our goal. Q.E.D.

The hypotheses of the following theorem are satisfied for compact injective spaces $Y$ with weight $\leqq 2^{\aleph_{0}}$ (hence for a product of at most $2^{\aleph_{0}}$ copies of $\left.[0,1]\right)$.

THeOREM 2. Let $X$ be a compact metric space and $Y$ an injective space of countable type whose points are intersections of at most $2^{N_{0}}$ open sets. Then $U(X, Y)$ is separable, hence of countable type. 
Proof. By [2, Chapter iii, Exercise 2], each countable uniform covering of $Y$ is uniform in a uniformly continuous pseudometric on $Y$ whose topology is separable. Therefore $Y$ is embeddable into a product $\prod_{\alpha \in A} Y_{\alpha}$ of separable metric spaces. Then $U(X, Y)$ is isomorphic to a subspace $Z$ of $\prod_{\alpha \in A} U\left(X, Y_{\alpha}\right)$. By [2, iii.25], $U(X, Y)$ is injective, so that there is a uniform retraction $r: \prod_{\alpha} U\left(X, Y_{\alpha}\right) \rightarrow Z$. Since $X$ is compact with a countable base, $U\left(X, Y_{\alpha}\right)=C_{c}\left(X, Y_{\alpha}\right)$ is separable by [3]. Then the above lemma implies the existence of $B \subseteq A$ such that $\operatorname{Card}(B) \leqq 2 \aleph_{0}$ and $r=g \circ \pi_{B}$ with $\pi_{B}$ the canonical projection $\prod_{\alpha \in A} U\left(X, Y_{\alpha}\right) \rightarrow \prod_{\alpha \in B} U\left(X, Y_{\alpha}\right)$ and $g: \prod_{\alpha \in B} U\left(X, Y_{\alpha}\right)$ $\rightarrow Z$ continuous. Since $\prod_{\alpha \in B} U\left(X, Y_{\alpha}\right)$ is separable by [1, viii.7.2.(3)] and since $g$ is onto, $Z$-hence $U(X, Y)$-must be separable. Q.E.D.

Corollary. An injective space $Y$ of countable type whose points are iniersections of at most $2^{\aleph_{0}}$ open sets must be separable. In particular, a compact injective space of weight $\leqq 2^{\aleph_{0}}$ must be separable.

Proof. The statement follows from Theorem 2 by taking $X$ reduced to a point. Q.E.D.

\section{REFERENCES}

1. J. Dugundji, Topology, Allyn and Bacon, Boston, Mass., 1966. MR 33 \#1824.

2. J. R. Isbell, Uniform spaces, Math. Surveys, no. 12, Amer. Math. Soc., Providence, R. I., 1964. MR $30 \# 561$.

3. E. Michael, On a theorem of Rudin and Klee, Proc. Amer. Math. Soc. 12 (1961), 921. MR 24 \#A2942.

4. G. Vidossich, Characterization of separability for LF-spaces, Ann. Inst. Fourier Grenoble 18 (1969), 87-90.

University of Pisa, Pisa, Italy 